\title{
Hippo inactivation feeds tumor-initiating cells
}

\author{
Stephan Duss, Adrian Britschgi and Mohamed Bentires-Alj*
}

\begin{abstract}
The Hippo pathway has emerged as a well-conserved kinase cascade controlling cell proliferation and survival and has recently gained much attention for its key activity as a tumor suppressor. In a study published in Cell, Cordenonsi and colleagues link TAZ, a downstream effector of the Hippo pathway, to attributes of putative breast cancer stem cells, epithelial-to-mesenchymal transition and cell polarity.
\end{abstract}

\section{Background}

The Hippo signaling pathway was discovered more than 17 years ago in Drosophila mutant screens as a major regulator of organ size controlling proliferation and cell death [1]. This pathway is conserved and equally important in mammals and, not surprisingly, deregulation of the Hippo pathway plays fundamental roles in cancer $[2,3]$. Whereas the upstream components of the Hippo pathway (NF2, MST1/2, SAV1, LATS1/2, and MOB1A) are tumor suppressors, the downstream components (YAP1, TAZ and TEAD) are oncogenes [4]. The mammalian Hippo homolog kinases MST1/2 (macrophage stimulating 1/2) in complex with the scaffold protein SAV1 (salvador homolog 1), phosphorylate and activate the kinases LATS1/2 (large tumor suppressor homolog $1 / 2$ ). LATS1/2 phosphorylate and inactivate the transcription co-activators YAP1 (Yes-associated protein 1) [5] and TAZ (transcriptional coactivator with PDZbinding motif) [6]. Unphosphorylated YAP1/TAZ associate with TEAD (TEA domain family member 1 ) and activate their target genes [7] (Figure 1). TAZ binds heteromeric SMAD2/3-4, activates transforming growth factor-beta target genes and maintains human embryonic stem cell self-renewal [8]. Furthermore, TAZ enhances epithelial-to-mesenchymal transition (EMT), migration, invasion and tumorigenesis of breast cancer cell lines $[6,9]$.

*Correspondence: bentires@fmi.ch

Friedrich Miescher Institute for Biomedical Research, Maulbeerstr. 66, 4058 Basel, Switzerland
Whereas many upstream components of the Hippo pathway in Drosophila [10] and zebrafish [11] have been identified, those in mammals remain poorly defined. The work of Cordenonsi and colleagues [12] indicates a role for EMT and the polarity gatekeeper Scribble upstream of the Hippo cascade in human breast tumor-initiating cells.

\section{The article}

To identify signaling pathways that drive breast tumorigenesis and heterogeneity, Cordenonsi and colleagues [12] analyzed a gene expression metadataset of breast tumors and found a TAZ/YAP signature to be correlated with high-grade (poorly differentiated) tumors. These tumors were previously shown to express embryonic and mammary stem cell signatures. Immunohistochemistry further revealed that TAZ is overexpressed in high-grade tumors, in part due to the amplification of the TAZencoding locus. The authors found that constitutive knockdown of TAZ in Ras-transformed MCF10A breast cancer models decreases tumorsphere formation capacity, the number of cells with the cancer stem cell phenotype $\left(\mathrm{CD} 44^{\text {High }} \mathrm{CD} 24^{\text {low }}\right)$, and tumorigenic potential. Expression of an activated mutant of TAZ in breast cancer cells increased the number of cells with the cancer stem cell phenotype, tumorsphere forming capacity, resistance to taxol, and the number of tumor-initiating cells.

The authors further assessed the relationship between EMT, tumorsphere formation and TAZ. They confirmed previous results showing that overexpression of TAZ induces EMT [6,9]. They also found that EMT increases TAZ protein expression and, notably, that TAZ increases tumorsphere formation independently of the loss of Ecadherin. Moreover, even though TAZ is a critical mediator of tumorsphere formation downstream of EMT, its inhibition was not sufficient for reversion of the EMT phenotype.

But how does EMT regulate TAZ? Scribble, a gatekeeper of epithelial cell polarity, is an upstream component of the Hippo pathway in zebrafish [11]. In breast cancer cells, Cordenonsi and colleagues were able to show that Scribble is essential for the nucleation of a complex of TAZ, LATS and MST. In particular, EMT causes delocalization of Scribble from the cell membrane, 


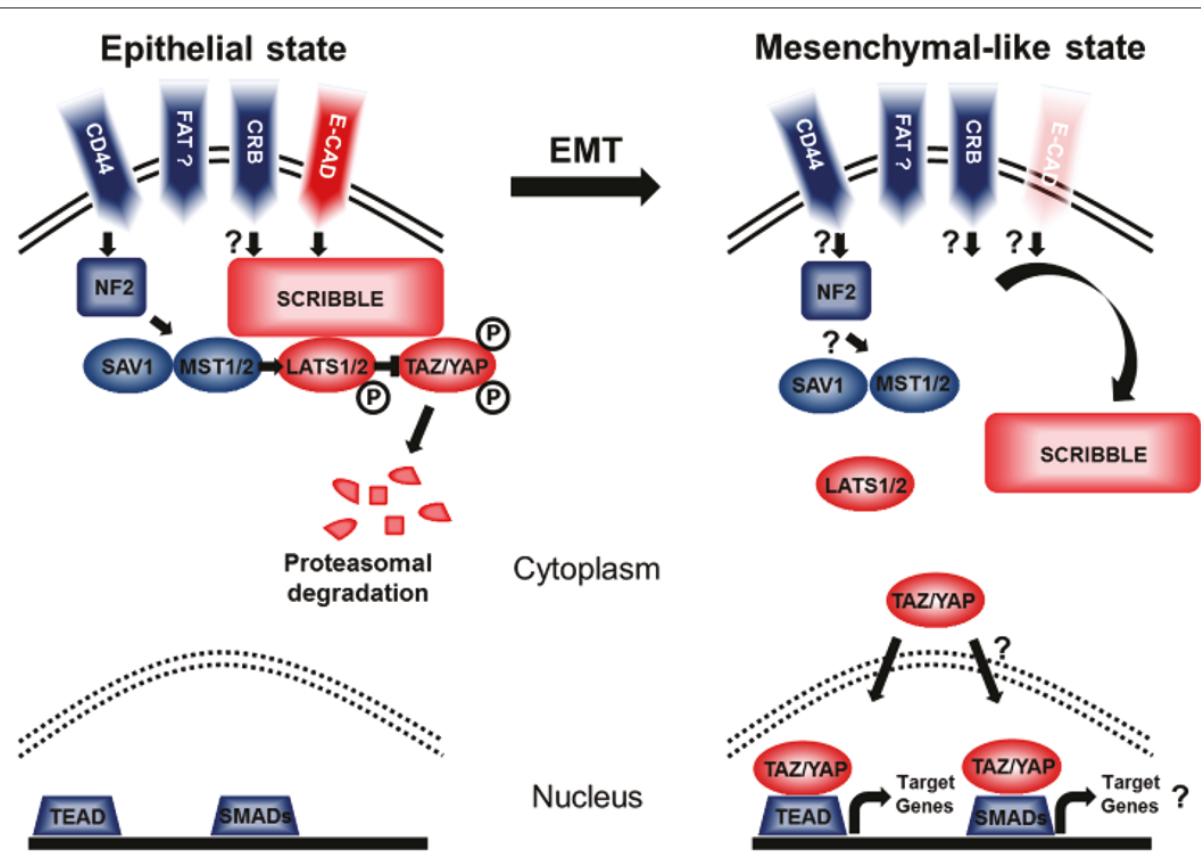

Figure 1. Activation of TAZ upon epithelial-to-mesenchymal transition in human breast cancer cells. A schematic of components of the mammalian Hippo pathway in epithelial (left) and mesenchymal-like (right) states of breast cancer cells. Components addressed by Cordenonsi and colleagues are shown in red. The authors demonstrated that Scribble is delocalized from the plasma membrane upon epithelial-to-mesenchymal transition (EMT), leading to stabilization of the TAZ protein and its translocation to the nucleus, where it activates downstream targets. Question marks indicate open questions.

which in turn relieves LATS-dependent TAZ inactivation and consequently induces TAZ stabilization and nuclear translocation (Figure 1, right).

\section{The viewpoint}

The paper by Cordenonsi and colleagues links Hippo signaling to EMT, cell polarity, tumorsphere formation, the cancer stem cell phenotype, and tumor-initiating cells. The fact that inactivation of Hippo signaling is crucial for a tumorigenic subpopulation of breast cancer cells raises the possibility that therapeutic activation of Hippo signaling or blockade of its downstream effectors could improve current treatment strategies. This is further underscored by the potent oncogenic activity of YAP in different preclinical cancer models and by the loss of function of LATS1/2 kinases in breast tumors [4].

The induction of metastases by TAZ activation was not reported by Cordenonsi and colleagues and warrants further investigation. It would also be important to address whether TAZ knockdown after overt tumor formation reduces tumor growth and progression.

Research in mammals is defining the upstream components of the Hippo pathway and some of them are linked to cell polarity, cell adhesion, and cell junction proteins [13]. The work of Cordenonsi and colleagues now adds Scribble to the list of mammalian upstream Hippo pathway components. Significantly, depletion of
Scribble in breast cells was shown previously to disrupt cell polarity, block three-dimensional morphogenesis, inhibit apoptosis, and induce dysplasia in vivo [14]. Other Hippo pathway upstream components such as NF2 (neurofibromin 2, merlin) [15,16], CD44 [15], the CRB (crumbs homolog) polarity complex [17], and the Ecadherin-catenin adhesion complex [18] also influence TAZ/YAP activity and EMT. For example, the CRB complex regulates TAZ/YAP and its disruption enhances transforming growth factor-beta signaling and activates SMAD-dependent transcription, which induces markers of EMT [17]. Hopefully, ongoing investigations will elucidate the interplay of these pathway components and identify specific upstream activators as well as downstream effectors in normal and neoplastic breast. These efforts should lead ultimately to the identification of novel targets for therapy.

\section{Abbreviations}

EMT, epithelial-to-mesenchymal transition.

\section{Competing interests}

The authors declare that they have no competing interests.

Published: 7 August 2012

\section{References}

1. Edgar BA: From cell structure to transcription: Hippo forges a new path. Cell 2006, 124:267-273.

2. Zeng $\mathrm{Q}$, Hong $\mathrm{W}$ : The emerging role of the hippo pathway in cell contact 
inhibition, organ size control, and cancer development in mammals. Cancer Cell 2008, 13:188-192.

3. Zhao B, Tumaneng K, Guan KL: The Hippo pathway in organ size control, tissue regeneration and stem cell self-renewal. Nat Cell Bio/ 2011, 13:877-883.

4. Chan SW, Lim CJ, Chen L, Chong YF, Huang C, Song H, Hong W: The Hippo pathway in biological control and cancer development. J Cell Physio/ 2011 226:928-939.

5. Hao Y, Chun A, Cheung K, Rashidi B, Yang X: Tumor suppressor LATS1 is a negative regulator of oncogene YAP. J Biol Chem 2008, 283:5496-5509.

6. Lei QY, Zhang H, Zhao B, Zha ZY, Bai F, Pei XH, Zhao S, Xiong Y, Guan KL: TAZ promotes cell proliferation and epithelial-mesenchymal transition and is inhibited by the hippo pathway. Mol Cell Biol 2008, 28:2426-2436.

7. Zhao B, Ye X, Yu J, Li L, Li W, Li S, Yu J, Lin JD, Wang CY, Chinnaiyan AM, Lai ZC, Guan KL: TEAD mediates YAP-dependent gene induction and growth control. Genes Dev 2008, 22:1962-1971.

8. Varelas X, Sakuma R, Samavarchi-Tehrani P, Peerani R, Rao BM, Dembowy J, Yaffe MB, Zandstra PW, Wrana JL: TAZ controls Smad nucleocytoplasmic shuttling and regulates human embryonic stem-cell self-renewal. Nat Cell Biol 2008, 10:837-848.

9. Chan SW, Lim CJ, Guo K, Ng CP, Lee I, Hunziker W, Zeng Q, Hong W: A role for TAZ in migration, invasion, and tumorigenesis of breast cancer cells. Cancer Res 2008, 68:2592-2598.

10. Grusche FA, Richardson HE, Harvey KF: Upstream regulation of the hippo size control pathway. Curr Biol 2010, 20:R574-582

11. Skouloudaki K, Puetz M, Simons M, Courbard JR, Boehlke C, Hartleben B, Engel C, Moeller MJ, Englert C, Bollig F, Schäfer T, Ramachandran H, Mlodzik M, Huber TB, Kuehn EW, Kim E, Kramer-Zucker A, Walz G: Scribble participates in Hippo signaling and is required for normal zebrafish pronephros development. Proc Natl Acad Sci U S A 2009, 106:8579-8584.
12. Cordenonsi M, Zanconato F, Azzolin L, Forcato M, Rosato A, Frasson C, Inui M Montagner M, Parenti AR, Poletti A, Daidone MG, Dupont S, Basso G, Bicciato S, Piccolo S: The Hippo transducer TAZ confers cancer stem cell-related traits on breast cancer cells. Cell 2011, 147:759-772.

13. Martin-Belmonte F, Perez-Moreno M: Epithelial cell polarity, stem cells and cancer. Nat Rev Cancer 2012, 12:23-38.

14. Zhan L, Rosenberg A, Bergami KC, Yu M, Xuan Z, Jaffe AB, Allred C, Muthuswamy SK: Deregulation of scribble promotes mammary tumorigenesis and reveals a role for cell polarity in carcinoma. Cell 2008, 135:865-878

15. Morrison H, Sherman LS, Legg J, Banine F, Isacke C, Haipek CA, Gutmann DH Ponta $\mathrm{H}$, Herrlich P: The NF2 tumor suppressor gene product, merlin, mediates contact inhibition of growth through interactions with CD44. Genes Dev 2001, 15:968-980.

16. Striedinger K, VandenBerg SR, Baia GS, McDermott MW, Gutmann DH, Lal A: The neurofibromatosis 2 tumor suppressor gene product, merlin, regulates human meningioma cell growth by signaling through YAP. Neoplasia 2008, 10:1204-1212.

17. Varelas X, Samavarchi-Tehrani P, Narimatsu M, Weiss A, Cockburn K, Larsen BG, Rossant J, Wrana JL: The Crumbs complex couples cell density sensing to Hippo-dependent control of the TGF-beta-SMAD pathway. Dev Cell 2010, 19:831-844.

18. Kim NG, Koh E, Chen X, Gumbiner BM: E-cadherin mediates contact inhibition of proliferation through Hippo signaling-pathway components. Proc Natl Acad Sci U S A 2011, 108:11930-11935.

doi: $10.1186 /$ bcr3190

Cite this article as: Duss $\mathrm{S}$, et al:: Hippo inactivation feeds tumor-initiating cells. Breast Cancer Research 2012, 14:318. 